\title{
A Comparison of Estimation Methods for Gompertz Flexible Weibull Distribution
}

\author{
Caner TANIŞ ${ }^{1}\left(\mathbb{D}\right.$, Kadir KARAKAYA ${ }^{2 *}$ \\ ${ }^{1}$ Çankırı Karatekin University, Faculty of Science, Department of Statistics, Çankırı, Turkey \\ ${ }^{2}$ Selçuk University, Faculty of Science, Department of Statistics, Konya, Turkey \\ Geliş / Received: 06/08/2020, Kabul / Accepted: 03/11/2021
}

\begin{abstract}
Gompertz flexible Weibull distribution is an extension of flexible Weibull distribution. We tackle the problem of the estimation of parameters of Gompertz flexible Weibull distribution. We discuss several methods of estimation for the Gompertz flexible Weibull distribution. The maximum likelihood estimators, least squares estimators, weighted least squares estimators, Anderson-Darling estimators, and Cramer-von Mises estimators are considered. A Monte Carlo simulation study is performed in order to compare these estimators in terms of their biases and mean square errors. According to the results of the simulation study, as the sample sizes increase the biases and mean square errors decrease in all parameter settings. Also, real data applications are presented to illustrate the usefulness of Gompertz flexible Weibull distribution and obtain estimates based on five methods of estimation.
\end{abstract}

Keywords: Gompertz flexible Weibull distribution, maximum likelihood estimators, least squares estimators, weighted least squares estimators.

\section{Gompertz Flexible Weibull Dağılımı için Tahmin Yöntemlerinin bir Karşılaştırması}

$\ddot{O} \mathbf{z}$

Gompertz flexible Weibull dağılımı, flexible Weibull dağılımının bir türüdür. Biz Gompertz flexible Weibull dağılımının parametrelerinin tahmini problemini ele aldık. Gompertz flexible Weibull dağılımı için birkaç tahmin yöntemi üzerinde durduk. En çok olabilirlik, en küçük kareler, ağırlıklandırılmış en küçük kareler, Anderson Darling ve Cramer-von Mises tahmin edicileri düşünülmüştür. Bu tahmin edicileri yan ve hata kareler ortalaması açısından karşılaştırabilmek için bir Monte Carlo simülasyon çalışması yapılmıştır. Simülasyon çalışmasının göre, tüm parametre durumlarında örneklem hacmi arttıkça yan ve hata kareler ortalaması azalmaktadır. Ayrıca Gompertz flexible Weibull dağılımının kullanışlılığını göstermek ve bahsedilen beş tahmin yöntemine dayalı olarak tahminleri elde etmek için gerçek veri çalışmaları sunulmuştur.

Anahtar Kelimeler: Gompertz flexible Weibull dağılımı, en çok olabilirlik tahmin edicisi, en küçük kareler tahmin edicisi, ağırlıklandırılmış en küçük kareler tahmin edicisi.

\section{Introduction}

Weibull distribution is a well-known lifetime distribution. It is widely used to determine the potential of wind energy. For instance, Kurban et al. (2007) examined the density of wind power and wind speed using Weibull distribution. Bebbington et al. (2007) proposed a new distribution as an alternative to Weibull distribution. Gompertz flexible Weibull distribution was introduced by Khaleel et al. (2020). It is an extension of flexible Weibull distribution suggested by Bebbington et al. (2007). We denote the Gompertz flexible Weibull distribution 
with four parameters as $\operatorname{GFW}(\alpha, \beta, \theta, \gamma)$. The probability density function (pdf) and the cumulative density function (cdf) of $\operatorname{GFW}(\alpha, \beta, \theta, \gamma)$ distribution are

$$
f(x)=\theta\left(\alpha+\frac{\beta}{x^{2}}\right) \exp \left(\alpha x-\frac{\beta}{X}\right) \times\left[\exp \left(-\exp \left(\alpha x-\frac{\beta}{x}\right)\right)\right]^{-\gamma} \times \exp \left(\frac{\theta}{\gamma}\left\{1-\left[\exp \left(-\exp \left(\alpha x-\frac{\beta}{X}\right)\right)\right]^{-\gamma}\right\}\right)
$$

and

$$
F(x)=1-\exp \left(\frac{\theta}{\gamma}\left\{1-\left[\exp \left(-\exp \left(\alpha x-\frac{\beta}{x}\right)\right)\right]^{-\gamma}\right\}\right)
$$

respectively, where $\alpha, \beta, \theta$ and $\gamma>0$. Khaleel et al. (2020) studied some characteristic properties of $\operatorname{GFW}(\alpha, \beta, \theta, \gamma)$ distribution. They illustrated the shape of the $\operatorname{GFW}(\alpha, \beta, \theta, \gamma)$ distribution could be decreasing or unimodal via plots at different values of parameters. Similarly, it can be concluded that the shape of the failure rate could be unimodal, increasing and decreasing according to graphs of hazard function (Khaleel et al., 2020). Thus it can be said that $\operatorname{GFW}(\alpha, \beta, \theta, \gamma)$ distribution is a very flexible distribution in data analysis. There is no study in literature since $\operatorname{GFW}(\alpha, \beta, \theta, \gamma)$ distribution is a fairly new proposed distribution. For this reason, we consider the estimation of parameters of $\operatorname{GFW}(\alpha, \beta, \theta, \gamma)$ distribution. The problem of parameter estimation is seen by many authors as a popular topic in recent years. There are many papers about different estimation methods for any distribution in the last decade. Asgharzadeh et al. (2011) discussed the methods of estimation for half-logistic distribution. Peng and Yan (2014) introduced a new extended Weibull distribution. They estimated the parameters of this new model using maximum likelihood estimators and Bayesian estimators in their study. Nassar et al. (2018) suggested a new distribution as an alternative Weibull distribution. They examined its properties and different estimation methods such as maximum likelihood, ordinary and weighted least squares, percentile and maximum product spacings in their paper. Tanış and Saraçoğlu (2019) compared six estimation methods for logKumaraswamy distribution. Ali et al. (2020a) considered a comparison of different methods of estimation for flexible Weibull distribution. Eliwa et al. (2020) focused the problem of point estimation for exponentiated odd Chen-G family of distributions. Ali et al. (2020b) provided a comparison of different methods of estimation for Logistic-Exponential distribution. Dey et al. (2020) studied the methods of point estimation for the parameters of weighted inverted Weibull distribution. Karakaya and Tanıs (2020a) compared the methods of estimation for the parameter of Akash distribution. Karakaya and Tanıs (2020b) discussed the methods of estimation for the parameters of Xgamma Weibull distribution. Tanış (2021) studied the estimation methods for transmuted power function distribution.

The main purpose of this paper is to estimate the parameters of the $\operatorname{GFW}(\alpha, \beta, \theta, \gamma)$ distribution using five different methods of estimation. Therefore, maximum likelihood estimators, least squares estimators, weighted least squares estimators, Anderson-Darling estimators and Crámer-von-Mises estimators are obtained for point estimation.

The rest of this paper is organized as follows: Section 2 describes five methods of estimation (maximum likelihood method, least squares method, weighted least squares method, the method of Anderson Darling, the method of Crámer-von-Mises). An extensive Monte Carlo 
simulation study is performed to compare these estimators according to mean square error (MSE) criteria in Section 3. In Section 4, it is considered real data illustrations. We also provide estimates of these estimators in real data analysis. Lastly, concluding remarks are given in Section 5 .

\section{Methods of Estimation}

In this section, five estimation methods are studied for estimating the unknown parameters of $\operatorname{GFW}(\alpha, \beta, \theta, \gamma)$ distribution. The maximum likelihood, least-squares introduced by Swain et al. (1988), weighted least squares introduced by Swain et al. (1988), Anderson-Darling introduced by Anderson and Darling (1952) and Crámer-von-Mises methods of estimation examined by Macdonald (1971) are investigated.

Let $X_{1}, X_{2}, \ldots, X_{n}$ be a random sample from the $\operatorname{GFW}(\alpha, \beta, \theta, \gamma)$ distribution. $X_{(1)}<X_{(2)}<\ldots<X_{(n)}$ symbolize the corresponding order statistics. Also, $X_{(i)}$ indicate the observed value of $X_{(i)}$ for $i=1,2, \ldots, n$. Then, the likelihood and log-likelihood function of the $\operatorname{GFW}(\alpha, \beta, \theta, \gamma)$ distribution are given, respectively, by

$$
L(\boldsymbol{\Xi})=\prod_{i=1}^{n} \theta\left(\alpha+\frac{\beta}{X_{i}^{2}}\right) \exp \left(\alpha x_{i}-\frac{\beta}{X_{i}}\right) \times\left[\exp \left(-\exp \left(\alpha x_{i}-\frac{\beta}{x_{i}}\right)\right)\right]^{-\gamma} \times \exp \left(\frac{\theta}{\gamma}\left\{1-\left[\exp \left(-\exp \left(\alpha x_{i}-\frac{\beta}{X_{i}}\right)\right)\right]^{-\gamma}\right\}\right)
$$

and

$$
\begin{aligned}
& \ell(\boldsymbol{\Xi})=n \log (\theta)+\sum_{i=1}^{n} \log \left(\alpha+\frac{\beta}{x_{i}^{2}}\right)+\sum_{i=1}^{n}\left(\alpha x_{i}-\frac{\beta}{X_{i}}\right)-\gamma \sum_{i=1}^{n}\left(-\exp \left(\alpha x_{i}-\frac{\beta}{x_{i}}\right)\right), \\
& +2 \sum_{i=1}^{n}\left(\frac{\theta}{\gamma}\left\{1-\left[\exp \left(-\exp \left(\alpha x_{i}-\frac{\beta}{X_{i}}\right)\right)\right]^{\gamma}\right\}\right)
\end{aligned}
$$

where $\boldsymbol{\Xi}=(\alpha, \beta, \theta, \gamma)$.

Then, the maximum likelihood estimator (MLE) of $\boldsymbol{\Xi}$ is given by

$$
\boldsymbol{\Xi}_{1}=\underset{\boldsymbol{\Xi}}{\arg \max }\{\ell(\boldsymbol{\Xi})\} \text {. }
$$

Let us define the following functions which are used to obtain the least-squares, weighted least squares, Anderson-Darling and Crámer-von-Mises estimates, respectively,

$$
Q_{L S}(\boldsymbol{\Xi})=\sum_{i=1}^{n}\left(\left\{1-\exp \left(\frac{\theta}{\gamma}\left\{1-\left[\exp \left(-\exp \left(\alpha X_{(i)}-\frac{\beta}{x_{(i)}}\right)\right)\right]^{-\gamma}\right\}\right)\right\}-\frac{i}{n+1}\right)^{2},
$$




$$
\begin{aligned}
& Q_{W L S}(\boldsymbol{\Xi})=\sum_{i=1}^{n} \frac{(n+2)(n+1)^{2}}{i(n-i+1)} \times\left(\left\{1-\exp \left(\frac{\theta}{\gamma}\left\{1-\left[\exp \left(-\exp \left(\alpha x_{(i)}-\frac{\beta}{X_{(i)}}\right)\right)\right]^{-\gamma}\right\}\right)\right\}-\frac{i}{n+1}\right)^{2}, \\
& \left.Q_{A D}(\boldsymbol{\Xi})=-n-\frac{1}{n} \sum_{i=1}^{n}\left[(2 i-1) \times \log \left(\left\{1-\exp \left(\frac{\theta}{\gamma}\left\{1-\left[\exp \left(-\exp \left(\alpha x_{(i)}-\frac{\beta}{x_{(i)}}\right)\right)\right]^{-\gamma}\right\}\right)\right)\right\}\right)\right] \\
& +\frac{1}{n} \sum_{i=1}^{n}\left(\operatorname { l o g } \left(\left\{\exp \left(\frac{\theta}{\gamma}\left\{1-\left[\exp \left(-\exp \left(\alpha x_{(i)}-\frac{\beta}{x_{(i)}}\right)\right)\right]\right\}\right)\right.\right.\right.
\end{aligned}
$$

and

$$
Q_{C V M}(\boldsymbol{\Xi})=\frac{1}{12 n}+\sum_{i=1}^{n}\left(\left\{1-\exp \left(\frac{\theta}{\gamma}\left\{1-\left[\exp \left(-\exp \left(\alpha x_{(i)}-\frac{\beta}{X_{(i)}}\right)\right)\right]^{-\gamma}\right\}\right)\right\}-\frac{2 i-1}{2 n}\right)^{2}
$$

Then, least square estimator (LSE), weighted least square estimator (WLSE), Anderson-Darling estimator (ADE) and Crámer-von-Mises estimator (CvME) of the parameter $\boldsymbol{\Xi}$ are given, respectively by

$$
\begin{aligned}
& \boldsymbol{\Xi}_{2}=\underset{\boldsymbol{\Xi}}{\arg \min }\left\{Q_{L S}(\boldsymbol{\Xi})\right\}, \\
& \boldsymbol{\Xi}_{3}=\underset{\boldsymbol{\Xi}}{\arg \min }\left\{Q_{W L S}(\boldsymbol{\Xi})\right\}, \\
& \boldsymbol{\Xi}_{4}=\underset{\boldsymbol{\Xi}}{\arg \min }\left\{Q_{A D}(\boldsymbol{\Xi})\right\}, \\
& \boldsymbol{\Xi}_{5}=\underset{\boldsymbol{\Xi}}{\arg \min }\left\{Q_{C V M}(\boldsymbol{\Xi})\right\}
\end{aligned}
$$

Five estimates given in (5)-(9) can be obtained by optim function in R with BFGS algorithm.

\section{Simulation Study}

In the simulation study, 5000 trials are conducted to estimate the biases and the MSEs of all estimators. Four true parameter settings are considered as $\boldsymbol{\Xi}=(0.25,0.25,025,0.25)(S 1)$, $(0.3,0.5,0.75,0.9)(S 2),(0.7,0.4,0.8,0.6)(S 3),(0.5,0.7,0.5,0.7)(S 4)$. The sample sizes are selected as $\mathrm{n}=(50,100,150,200,250,300,350,400,450,500)$. The BFGS algorithm which is available in $\mathrm{R}$ is used to achieve five estimates given in (5)-(9). In Tables 1-5, biases and MSEs of MLE, LSE, WLSE, ADE and CVME are reported. Tables 1-5 indicate that the biases and MSEs of 
five estimators are close to zero when the sample size increases as expected. Also, the MSEs of all estimators are bigger than the others for the parameter $\gamma$. It can be concluded that the MLE and WLSE generally have the smallest MSE for all parameters.

Table 1: Average biases and MSEs of MLE

\begin{tabular}{|c|c|c|c|c|c|c|c|c|c|}
\hline \multicolumn{5}{|c|}{ Bias } & \multicolumn{5}{|c|}{ MSEs } \\
\hline & $n$ & $\alpha$ & $\beta$ & $\theta$ & $\gamma$ & $\alpha$ & $\beta$ & $\theta$ & $\gamma$ \\
\hline \multirow{10}{*}{$\mathrm{S} 1$} & 50 & -0.0073 & 0.0178 & -0.0222 & 0.2259 & 0.0060 & 0.0112 & 0.0056 & 0.3389 \\
\hline & 100 & -0.0058 & 0.0016 & -0.0238 & 0.2080 & 0.0056 & 0.0046 & 0.0037 & 0.3139 \\
\hline & 150 & -0.0028 & -0.0022 & -0.0236 & 0.1791 & 0.0051 & 0.0027 & 0.0029 & 0.2739 \\
\hline & 200 & -0.0043 & -0.0039 & -0.0221 & 0.1707 & 0.0047 & 0.0020 & 0.0024 & 0.2386 \\
\hline & 250 & -0.0068 & -0.0049 & -0.0213 & 0.1740 & 0.0044 & 0.0016 & 0.0022 & 0.2321 \\
\hline & 300 & -0.0061 & -0.0049 & -0.0200 & 0.1604 & 0.0041 & 0.0014 & 0.0019 & 0.2057 \\
\hline & 350 & -0.0058 & -0.0059 & -0.0194 & 0.1516 & 0.0038 & 0.0012 & 0.0018 & 0.1883 \\
\hline & 400 & -0.0065 & -0.0051 & -0.0173 & 0.1408 & 0.0035 & 0.0010 & 0.0015 & 0.1640 \\
\hline & 450 & -0.0057 & -0.0045 & -0.0162 & 0.1309 & 0.0033 & 0.0009 & 0.0014 & 0.1490 \\
\hline & 500 & -0.0070 & -0.0047 & -0.0170 & 0.1360 & 0.0033 & 0.0008 & 0.0013 & 0.1488 \\
\hline \multirow{10}{*}{$\mathrm{S} 2$} & 50 & 0.0553 & -0.0034 & -0.0281 & 0.2179 & 0.0286 & 0.0238 & 0.1342 & 1.6467 \\
\hline & 100 & 0.0399 & -0.0147 & -0.0497 & 0.2056 & 0.0226 & 0.0136 & 0.0799 & 1.2926 \\
\hline & 150 & 0.0323 & -0.0175 & -0.0548 & 0.2052 & 0.0203 & 0.0107 & 0.0666 & 1.1734 \\
\hline & 200 & 0.0349 & -0.0150 & -0.0459 & 0.1380 & 0.0191 & 0.0079 & 0.0522 & 0.9012 \\
\hline & 250 & 0.0337 & -0.0159 & -0.0480 & 0.1252 & 0.0184 & 0.0067 & 0.0444 & 0.7977 \\
\hline & 300 & 0.0329 & -0.0108 & -0.0360 & 0.0878 & 0.0167 & 0.0054 & 0.0383 & 0.6602 \\
\hline & 350 & 0.0340 & -0.0120 & -0.0400 & 0.0764 & 0.0167 & 0.0048 & 0.0343 & 0.6037 \\
\hline & 400 & 0.0341 & -0.0114 & -0.0360 & 0.0594 & 0.0161 & 0.0042 & 0.0311 & 0.5326 \\
\hline & 450 & 0.0348 & -0.0079 & -0.0291 & 0.0412 & 0.0158 & 0.0038 & 0.0284 & 0.4929 \\
\hline & 500 & 0.0314 & -0.0092 & -0.0294 & 0.0421 & 0.0147 & 0.0034 & 0.0266 & 0.4461 \\
\hline \multirow{10}{*}{ S3 } & 50 & 0.0003 & -0.0120 & -0.0643 & 0.4554 & 0.0933 & 0.0145 & 0.1605 & 1.6945 \\
\hline & 100 & -0.0183 & -0.0203 & -0.0948 & 0.4496 & 0.0784 & 0.0084 & 0.0878 & 1.4882 \\
\hline & 150 & -0.0205 & -0.0197 & -0.0910 & 0.4032 & 0.0719 & 0.0061 & 0.0661 & 1.2593 \\
\hline & 200 & -0.0224 & -0.0200 & -0.0904 & 0.3747 & 0.0686 & 0.0049 & 0.0559 & 1.0588 \\
\hline & 250 & -0.0116 & -0.0173 & -0.0798 & 0.3008 & 0.0617 & 0.0037 & 0.0436 & 0.8029 \\
\hline & 300 & -0.0098 & -0.0162 & -0.0772 & 0.2802 & 0.0605 & 0.0031 & 0.0382 & 0.7152 \\
\hline & 350 & -0.0156 & -0.0156 & -0.0735 & 0.2725 & 0.0564 & 0.0027 & 0.0340 & 0.6470 \\
\hline & 400 & -0.0047 & -0.0135 & -0.0661 & 0.2245 & 0.0539 & 0.0022 & 0.0278 & 0.5175 \\
\hline & 450 & -0.0132 & -0.0137 & -0.0668 & 0.2372 & 0.0526 & 0.0020 & 0.0264 & 0.5037 \\
\hline & 500 & -0.0098 & -0.0125 & -0.0645 & 0.2220 & 0.0516 & 0.0018 & 0.0239 & 0.4574 \\
\hline \multirow{10}{*}{ S4 } & 50 & 0.0329 & -0.0004 & -0.0272 & 0.3198 & 0.0448 & 0.0624 & 0.0747 & 1.4437 \\
\hline & 100 & 0.0071 & -0.0244 & -0.0459 & 0.3488 & 0.0356 & 0.0338 & 0.0405 & 1.2934 \\
\hline & 150 & -0.0004 & -0.0268 & -0.0511 & 0.3401 & 0.0327 & 0.0237 & 0.0289 & 1.1370 \\
\hline & 200 & -0.0070 & -0.0285 & -0.0522 & 0.3314 & 0.0306 & 0.0191 & 0.0243 & 0.9774 \\
\hline & 250 & -0.0012 & -0.0270 & -0.0494 & 0.2817 & 0.0288 & 0.0149 & 0.0197 & 0.8284 \\
\hline & 300 & 0.0002 & -0.0276 & -0.0496 & 0.2633 & 0.0282 & 0.0132 & 0.0177 & 0.7595 \\
\hline & 350 & -0.0052 & -0.0264 & -0.0469 & 0.2516 & 0.0244 & 0.0113 & 0.0154 & 0.6512 \\
\hline & 400 & -0.0061 & -0.0253 & -0.0451 & 0.2412 & 0.0233 & 0.0100 & 0.0140 & 0.5972 \\
\hline & 450 & -0.0036 & -0.0234 & -0.0408 & 0.2087 & 0.0215 & 0.0086 & 0.0121 & 0.5071 \\
\hline & 500 & -0.0026 & -0.0216 & -0.0396 & 0.2015 & 0.0216 & 0.0079 & 0.0112 & 0.4865 \\
\hline
\end{tabular}


Table 2: Average biases and MSEs of LSE

\begin{tabular}{|c|c|c|c|c|c|c|c|c|c|}
\hline \multicolumn{5}{|c|}{ Bias } & \multicolumn{5}{|c|}{ MSEs } \\
\hline & $n$ & $\alpha$ & $\beta$ & $\theta$ & $\gamma$ & $\alpha$ & $\beta$ & $\theta$ & $\gamma$ \\
\hline \multirow{10}{*}{ S1 } & 50 & -0.0016 & -0.0248 & -0.0225 & 0.2198 & 0.0111 & 0.0157 & 0.0059 & 0.2489 \\
\hline & 100 & 0.0004 & -0.0204 & -0.0219 & 0.1729 & 0.0083 & 0.0069 & 0.0032 & 0.1867 \\
\hline & 150 & 0.0032 & -0.0179 & -0.0216 & 0.1413 & 0.0070 & 0.0043 & 0.0024 & 0.1550 \\
\hline & 200 & 0.0006 & -0.0161 & -0.0195 & 0.1326 & 0.0060 & 0.0031 & 0.0019 & 0.1340 \\
\hline & 250 & -0.0016 & -0.0142 & -0.0177 & 0.1269 & 0.0052 & 0.0025 & 0.0016 & 0.1226 \\
\hline & 300 & -0.0009 & -0.0134 & -0.0170 & 0.1175 & 0.0048 & 0.0021 & 0.0014 & 0.1116 \\
\hline & 350 & -0.0001 & -0.0128 & -0.0158 & 0.1056 & 0.0044 & 0.0018 & 0.0012 & 0.1023 \\
\hline & 400 & -0.0015 & -0.0118 & -0.0147 & 0.1038 & 0.0041 & 0.0016 & 0.0011 & 0.0969 \\
\hline & 450 & -0.0010 & -0.0108 & -0.0136 & 0.0946 & 0.0037 & 0.0014 & 0.0010 & 0.0869 \\
\hline & 500 & -0.0018 & -0.0101 & -0.0140 & 0.0971 & 0.0036 & 0.0013 & 0.0009 & 0.0866 \\
\hline \multirow{10}{*}{ S2 } & 50 & 0.0103 & -0.0398 & -0.0406 & 0.4084 & 0.0383 & 0.0338 & 0.2490 & 1.8042 \\
\hline & 100 & 0.0082 & -0.0536 & -0.1047 & 0.4817 & 0.0347 & 0.0210 & 0.1411 & 1.8508 \\
\hline & 150 & 0.0101 & -0.0487 & -0.1023 & 0.4363 & 0.0316 & 0.0170 & 0.1173 & 1.6815 \\
\hline & 200 & 0.0160 & -0.0436 & -0.0926 & 0.3597 & 0.0295 & 0.0138 & 0.0968 & 1.4332 \\
\hline & 250 & 0.0195 & -0.0416 & -0.0920 & 0.3211 & 0.0283 & 0.0118 & 0.0826 & 1.2838 \\
\hline & 300 & 0.0202 & -0.0338 & -0.0764 & 0.2731 & 0.0267 & 0.0102 & 0.0740 & 1.1365 \\
\hline & 350 & 0.0210 & -0.0347 & -0.0812 & 0.2591 & 0.0252 & 0.0091 & 0.0671 & 1.0573 \\
\hline & 400 & 0.0272 & -0.0308 & -0.0700 & 0.2063 & 0.0249 & 0.0082 & 0.0605 & 0.9608 \\
\hline & 450 & 0.0236 & -0.0289 & -0.0693 & 0.2097 & 0.0240 & 0.0076 & 0.0568 & 0.8998 \\
\hline & 500 & 0.0247 & -0.0275 & -0.0643 & 0.1867 & 0.0231 & 0.0069 & 0.0526 & 0.8460 \\
\hline \multirow{10}{*}{ S3 } & 50 & -0.0928 & -0.0303 & -0.0225 & 0.5587 & 0.1250 & 0.0208 & 0.3166 & 1.6901 \\
\hline & 100 & -0.0852 & -0.0417 & -0.1117 & 0.6328 & 0.1192 & 0.0128 & 0.1461 & 1.7784 \\
\hline & 150 & -0.0791 & -0.0419 & -0.1247 & 0.6236 & 0.1153 & 0.0100 & 0.1161 & 1.7638 \\
\hline & 200 & -0.0700 & -0.0437 & -0.1382 & 0.6148 & 0.1184 & 0.0086 & 0.1029 & 1.6890 \\
\hline & 250 & -0.0707 & -0.0410 & -0.1307 & 0.5667 & 0.1021 & 0.0072 & 0.0871 & 1.4876 \\
\hline & 300 & -0.0681 & -0.0376 & -0.1241 & 0.5313 & 0.0955 & 0.0062 & 0.0760 & 1.3390 \\
\hline & 350 & -0.0671 & -0.0355 & -0.1182 & 0.5058 & 0.0893 & 0.0056 & 0.0704 & 1.2525 \\
\hline & 400 & -0.0574 & -0.0331 & -0.1107 & 0.4584 & 0.0851 & 0.0050 & 0.0619 & 1.1149 \\
\hline & 450 & -0.0627 & -0.0323 & -0.1108 & 0.4618 & 0.0816 & 0.0046 & 0.0585 & 1.0766 \\
\hline & 500 & -0.0591 & -0.0302 & -0.1073 & 0.4424 & 0.0805 & 0.0042 & 0.0537 & 1.0020 \\
\hline \multirow{10}{*}{ S4 } & 50 & -0.0576 & -0.0438 & 0.0094 & 0.4409 & 0.0374 & 0.0941 & 0.1619 & 1.0596 \\
\hline & 100 & -0.0581 & -0.0668 & -0.0504 & 0.5173 & 0.0377 & 0.0513 & 0.0640 & 1.2106 \\
\hline & 150 & -0.0567 & -0.0726 & -0.0709 & 0.5452 & 0.0383 & 0.0375 & 0.0466 & 1.2844 \\
\hline & 200 & -0.0703 & -0.0829 & -0.0881 & 0.6230 & 0.0368 & 0.0325 & 0.0422 & 1.4294 \\
\hline & 250 & -0.0694 & -0.0788 & -0.0863 & 0.5996 & 0.0342 & 0.0285 & 0.0378 & 1.3413 \\
\hline & 300 & -0.0644 & -0.0775 & -0.0870 & 0.5698 & 0.0328 & 0.0250 & 0.0338 & 1.2723 \\
\hline & 350 & -0.0613 & -0.0771 & -0.0876 & 0.5527 & 0.0321 & 0.0224 & 0.0310 & 1.2252 \\
\hline & 400 & -0.0568 & -0.0698 & -0.0797 & 0.5070 & 0.0300 & 0.0201 & 0.0280 & 1.0948 \\
\hline & 450 & -0.0589 & -0.0693 & -0.0787 & 0.4967 & 0.0284 & 0.0183 & 0.0258 & 1.0222 \\
\hline & 500 & -0.0551 & -0.0647 & -0.0756 & 0.4781 & 0.0289 & 0.0168 & 0.0241 & 0.9867 \\
\hline
\end{tabular}


Table 3: Average biases and MSEs of WLSE

\begin{tabular}{|c|c|c|c|c|c|c|c|c|c|}
\hline \multicolumn{5}{|c|}{ Bias } & \multicolumn{5}{|c|}{ MSEs } \\
\hline & $n$ & $\alpha$ & $\beta$ & $\theta$ & $\gamma$ & $\alpha$ & $\beta$ & $\theta$ & $\gamma$ \\
\hline \multirow{10}{*}{ S1 } & 50 & 0.0041 & -0.0182 & -0.0193 & 0.1706 & 0.0093 & 0.0108 & 0.0054 & 0.2237 \\
\hline & 100 & 0.0047 & -0.0125 & -0.0165 & 0.1167 & 0.0065 & 0.0051 & 0.0027 & 0.1329 \\
\hline & 150 & 0.0071 & -0.0105 & -0.0160 & 0.0878 & 0.0054 & 0.0030 & 0.0020 & 0.1040 \\
\hline & 200 & 0.0044 & -0.0094 & -0.0144 & 0.0830 & 0.0046 & 0.0021 & 0.0015 & 0.0866 \\
\hline & 250 & 0.0026 & -0.0078 & -0.0121 & 0.0735 & 0.0038 & 0.0017 & 0.0012 & 0.0717 \\
\hline & 300 & 0.0034 & -0.0071 & -0.0115 & 0.0658 & 0.0035 & 0.0014 & 0.0010 & 0.0656 \\
\hline & 350 & 0.0035 & -0.0074 & -0.0109 & 0.0592 & 0.0032 & 0.0012 & 0.0009 & 0.0600 \\
\hline & 400 & 0.0024 & -0.0064 & -0.0094 & 0.0555 & 0.0029 & 0.0010 & 0.0007 & 0.0538 \\
\hline & 450 & 0.0028 & -0.0056 & -0.0088 & 0.0503 & 0.0027 & 0.0009 & 0.0007 & 0.0492 \\
\hline & 500 & 0.0020 & -0.0052 & -0.0092 & 0.0520 & 0.0026 & 0.0008 & 0.0006 & 0.0486 \\
\hline \multirow{10}{*}{ S2 } & 50 & 0.0134 & -0.0622 & -0.1097 & 0.6533 & 0.0466 & 0.0358 & 0.2342 & 2.8505 \\
\hline & 100 & 0.0101 & -0.0529 & -0.1138 & 0.5087 & 0.0314 & 0.0198 & 0.1278 & 2.0197 \\
\hline & 150 & 0.0186 & -0.0415 & -0.0951 & 0.3800 & 0.0308 & 0.0144 & 0.0974 & 1.5397 \\
\hline & 200 & 0.0191 & -0.0335 & -0.0769 & 0.2853 & 0.0243 & 0.0108 & 0.0761 & 1.1883 \\
\hline & 250 & 0.0238 & -0.0304 & -0.0727 & 0.2334 & 0.0238 & 0.0087 & 0.0622 & 1.0097 \\
\hline & 300 & 0.0247 & -0.0239 & -0.0593 & 0.1893 & 0.0226 & 0.0072 & 0.0540 & 0.8641 \\
\hline & 350 & 0.0257 & -0.0232 & -0.0596 & 0.1638 & 0.0211 & 0.0062 & 0.0476 & 0.7608 \\
\hline & 400 & 0.0298 & -0.0202 & -0.0504 & 0.1225 & 0.0204 & 0.0054 & 0.0423 & 0.6802 \\
\hline & 450 & 0.0296 & -0.0162 & -0.0428 & 0.0996 & 0.0194 & 0.0047 & 0.0374 & 0.5977 \\
\hline & 500 & 0.0278 & -0.0165 & -0.0416 & 0.0948 & 0.0184 & 0.0043 & 0.0347 & 0.5648 \\
\hline \multirow{10}{*}{ S3 } & 50 & -0.0565 & -0.0574 & -0.1396 & 0.8485 & 0.1964 & 0.0231 & 0.2785 & 3.1568 \\
\hline & 100 & -0.0730 & -0.0486 & -0.1522 & 0.7362 & 0.1268 & 0.0127 & 0.1406 & 2.3497 \\
\hline & 150 & -0.0526 & -0.0375 & -0.1256 & 0.5755 & 0.1130 & 0.0084 & 0.0977 & 1.7253 \\
\hline & 200 & -0.0572 & -0.0353 & -0.1226 & 0.5379 & 0.0992 & 0.0068 & 0.0816 & 1.4579 \\
\hline & 250 & -0.0527 & -0.0305 & -0.1078 & 0.4552 & 0.0817 & 0.0051 & 0.0637 & 1.1318 \\
\hline & 300 & -0.0378 & -0.0266 & -0.0981 & 0.3955 & 0.0824 & 0.0042 & 0.0532 & 0.9646 \\
\hline & 350 & -0.0378 & -0.0246 & -0.0918 & 0.3696 & 0.0763 & 0.0036 & 0.0474 & 0.8659 \\
\hline & 400 & -0.0282 & -0.0220 & -0.0828 & 0.3202 & 0.0716 & 0.0031 & 0.0397 & 0.7347 \\
\hline & 450 & -0.0377 & -0.0216 & -0.0832 & 0.3310 & 0.0672 & 0.0028 & 0.0371 & 0.7015 \\
\hline & 500 & -0.0415 & -0.0197 & -0.0789 & 0.3222 & 0.0611 & 0.0025 & 0.0338 & 0.6444 \\
\hline \multirow{10}{*}{ S4 } & 50 & -0.0040 & -0.1028 & -0.0859 & 0.6530 & 0.0956 & 0.0871 & 0.1183 & 2.2215 \\
\hline & 100 & -0.0328 & -0.0888 & -0.0920 & 0.6165 & 0.0561 & 0.0470 & 0.0591 & 1.8434 \\
\hline & 150 & -0.0325 & -0.0736 & -0.0852 & 0.5412 & 0.0479 & 0.0319 & 0.0415 & 1.5071 \\
\hline & 200 & -0.0462 & -0.0704 & -0.0839 & 0.5453 & 0.0386 & 0.0268 & 0.0360 & 1.3725 \\
\hline & 250 & -0.0408 & -0.0612 & -0.0750 & 0.4779 & 0.0347 & 0.0209 & 0.0292 & 1.1482 \\
\hline & 300 & -0.0335 & -0.0557 & -0.0702 & 0.4209 & 0.0327 & 0.0178 & 0.0251 & 0.9833 \\
\hline & 350 & -0.0347 & -0.0533 & -0.0677 & 0.4039 & 0.0297 & 0.0155 & 0.0222 & 0.9062 \\
\hline & 400 & -0.0311 & -0.0469 & -0.0603 & 0.3596 & 0.0274 & 0.0130 & 0.0191 & 0.7695 \\
\hline & 450 & -0.0336 & -0.0459 & -0.0580 & 0.3470 & 0.0244 & 0.0119 & 0.0174 & 0.7078 \\
\hline & 500 & -0.0305 & -0.0410 & -0.0537 & 0.3257 & 0.0239 & 0.0105 & 0.0156 & 0.6646 \\
\hline
\end{tabular}


Table 4: Average biases and MSEs of ADE

\begin{tabular}{|c|c|c|c|c|c|c|c|c|c|}
\hline \multicolumn{5}{|c|}{ Bias } & \multicolumn{5}{|c|}{ MSEs } \\
\hline & $n$ & $\alpha$ & $\beta$ & $\theta$ & $\gamma$ & $\alpha$ & $\beta$ & $\theta$ & $\gamma$ \\
\hline \multirow{10}{*}{ S } & 50 & -0.0002 & -0.0109 & -0.0260 & 0.2404 & 0.0100 & 0.0098 & 0.0062 & 0.3576 \\
\hline & 100 & 0.0003 & -0.0123 & -0.0240 & 0.1879 & 0.0076 & 0.0047 & 0.0034 & 0.2456 \\
\hline & 150 & 0.0020 & -0.0120 & -0.0234 & 0.1593 & 0.0065 & 0.0029 & 0.0027 & 0.2094 \\
\hline & 200 & -0.0012 & -0.0116 & -0.0217 & 0.1546 & 0.0057 & 0.0021 & 0.0021 & 0.1787 \\
\hline & 250 & -0.0039 & -0.0106 & -0.0197 & 0.1493 & 0.0049 & 0.0017 & 0.0018 & 0.1625 \\
\hline & 300 & -0.0037 & -0.0102 & -0.0193 & 0.1446 & 0.0047 & 0.0015 & 0.0016 & 0.1565 \\
\hline & 350 & -0.0036 & -0.0105 & -0.0184 & 0.1360 & 0.0043 & 0.0013 & 0.0015 & 0.1451 \\
\hline & 400 & -0.0050 & -0.0098 & -0.0172 & 0.1343 & 0.0041 & 0.0011 & 0.0013 & 0.1390 \\
\hline & 450 & -0.0048 & -0.0088 & -0.0162 & 0.1271 & 0.0038 & 0.0010 & 0.0013 & 0.1292 \\
\hline & 500 & -0.0061 & -0.0086 & -0.0169 & 0.1325 & 0.0037 & 0.0009 & 0.0012 & 0.1300 \\
\hline \multirow{10}{*}{ S2 } & 50 & 0.0073 & -0.0446 & -0.0845 & 0.6211 & 0.0347 & 0.0315 & 0.2174 & 2.7613 \\
\hline & 100 & 0.0071 & -0.0443 & -0.1022 & 0.4956 & 0.0282 & 0.0183 & 0.1209 & 1.9435 \\
\hline & 150 & 0.0103 & -0.0376 & -0.0917 & 0.4011 & 0.0261 & 0.0138 & 0.0957 & 1.5350 \\
\hline & 200 & 0.0159 & -0.0313 & -0.0760 & 0.3019 & 0.0237 & 0.0104 & 0.0757 & 1.1993 \\
\hline & 250 & 0.0172 & -0.0294 & -0.0740 & 0.2630 & 0.0219 & 0.0087 & 0.0629 & 1.0298 \\
\hline & 300 & 0.0173 & -0.0234 & -0.0614 & 0.2218 & 0.0203 & 0.0073 & 0.0553 & 0.8898 \\
\hline & 350 & 0.0188 & -0.0232 & -0.0628 & 0.1961 & 0.0194 & 0.0063 & 0.0487 & 0.7820 \\
\hline & 400 & 0.0241 & -0.0205 & -0.0536 & 0.1512 & 0.0192 & 0.0055 & 0.0433 & 0.6989 \\
\hline & 450 & 0.0235 & -0.0169 & -0.0471 & 0.1324 & 0.0183 & 0.0048 & 0.0390 & 0.6244 \\
\hline & 500 & 0.0218 & -0.0173 & -0.0461 & 0.1268 & 0.0173 & 0.0044 & 0.0360 & 0.5867 \\
\hline \multirow{10}{*}{ S3 } & 50 & -0.1011 & -0.0460 & -0.1221 & 0.9068 & 0.1404 & 0.0201 & 0.2632 & 3.3053 \\
\hline & 100 & -0.0845 & -0.0423 & -0.1407 & 0.7392 & 0.1148 & 0.0116 & 0.1332 & 2.3064 \\
\hline & 150 & -0.0417 & -0.0334 & -0.1184 & 0.5475 & 0.1138 & 0.0080 & 0.0945 & 1.6658 \\
\hline & 200 & -0.0413 & -0.0317 & -0.1161 & 0.5015 & 0.1051 & 0.0065 & 0.0783 & 1.3951 \\
\hline & 250 & -0.0558 & -0.0292 & -0.1064 & 0.4678 & 0.0831 & 0.0050 & 0.0639 & 1.1563 \\
\hline & 300 & -0.0521 & -0.0260 & -0.0985 & 0.4273 & 0.0777 & 0.0042 & 0.0540 & 1.0021 \\
\hline & 350 & -0.0525 & -0.0245 & -0.0938 & 0.4060 & 0.0725 & 0.0037 & 0.0489 & 0.9154 \\
\hline & 400 & -0.0467 & -0.0221 & -0.0849 & 0.3616 & 0.0660 & 0.0032 & 0.0413 & 0.7808 \\
\hline & 450 & -0.0515 & -0.0218 & -0.0855 & 0.3653 & 0.0646 & 0.0028 & 0.0386 & 0.7422 \\
\hline & 500 & -0.0490 & -0.0198 & -0.0812 & 0.3464 & 0.0618 & 0.0025 & 0.0348 & 0.6801 \\
\hline \multirow{10}{*}{ S4 } & 50 & -0.0538 & -0.0825 & -0.0788 & 0.8128 & 0.0638 & 0.0811 & 0.1180 & 2.6592 \\
\hline & 100 & -0.0150 & -0.0744 & -0.0833 & 0.5629 & 0.0605 & 0.0444 & 0.0573 & 1.8098 \\
\hline & 150 & -0.0425 & -0.0692 & -0.0841 & 0.5834 & 0.0456 & 0.0314 & 0.0417 & 1.5879 \\
\hline & 200 & -0.0550 & -0.0682 & -0.0842 & 0.5879 & 0.0376 & 0.0270 & 0.0370 & 1.4711 \\
\hline & 250 & -0.0492 & -0.0599 & -0.0762 & 0.5174 & 0.0343 & 0.0214 & 0.0300 & 1.2208 \\
\hline & 300 & -0.0477 & -0.0572 & -0.0738 & 0.4826 & 0.0313 & 0.0186 & 0.0266 & 1.0851 \\
\hline & 350 & -0.0472 & -0.0553 & -0.0715 & 0.4614 & 0.0291 & 0.0163 & 0.0236 & 0.9996 \\
\hline & 400 & -0.0402 & -0.0488 & -0.0642 & 0.4073 & 0.0277 & 0.0139 & 0.0204 & 0.8564 \\
\hline & 450 & -0.0374 & -0.0475 & -0.0614 & 0.3789 & 0.0263 & 0.0125 & 0.0185 & 0.7744 \\
\hline & 500 & -0.0340 & -0.0427 & -0.0573 & 0.3562 & 0.0261 & 0.0111 & 0.0167 & 0.7268 \\
\hline
\end{tabular}


Table 5: Average biases and MSEs of CVME

\begin{tabular}{|c|c|c|c|c|c|c|c|c|c|}
\hline \multicolumn{5}{|c|}{ Bias } & \multicolumn{5}{|c|}{ MSEs } \\
\hline & $n$ & $\alpha$ & $\beta$ & $\theta$ & $\gamma$ & $\alpha$ & $\beta$ & $\theta$ & $\gamma$ \\
\hline \multirow{10}{*}{$S$} & 50 & -0.0029 & -0.0016 & -0.0220 & 0.2225 & 0.0105 & 0.0186 & 0.0059 & 0.2219 \\
\hline & 100 & -0.0009 & -0.0089 & -0.0221 & 0.1777 & 0.0080 & 0.0073 & 0.0032 & 0.1756 \\
\hline & 150 & 0.0020 & -0.0103 & -0.0218 & 0.1463 & 0.0068 & 0.0044 & 0.0024 & 0.1490 \\
\hline & 200 & -0.0002 & -0.0104 & -0.0196 & 0.1353 & 0.0058 & 0.0031 & 0.0019 & 0.1270 \\
\hline & 250 & -0.0025 & -0.0098 & -0.0179 & 0.1313 & 0.0051 & 0.0025 & 0.0016 & 0.1198 \\
\hline & 300 & -0.0017 & -0.0097 & -0.0173 & 0.1214 & 0.0047 & 0.0021 & 0.0014 & 0.1090 \\
\hline & 350 & -0.0008 & -0.0096 & -0.0160 & 0.1088 & 0.0043 & 0.0018 & 0.0012 & 0.0996 \\
\hline & 400 & -0.0021 & -0.0090 & -0.0148 & 0.1066 & 0.0040 & 0.0015 & 0.0011 & 0.0942 \\
\hline & 450 & -0.0017 & -0.0083 & -0.0138 & 0.0983 & 0.0037 & 0.0014 & 0.0010 & 0.0857 \\
\hline & 500 & -0.0024 & -0.0078 & -0.0142 & 0.0998 & 0.0036 & 0.0013 & 0.0009 & 0.0851 \\
\hline \multirow{10}{*}{ S2 } & 50 & 0.0356 & -0.0029 & 0.0268 & 0.2250 & 0.0382 & 0.0337 & 0.2633 & 1.4909 \\
\hline & 100 & 0.0262 & -0.0316 & -0.0632 & 0.3458 & 0.0351 & 0.0192 & 0.1336 & 1.6108 \\
\hline & 150 & 0.0243 & -0.0326 & -0.0717 & 0.3300 & 0.0319 & 0.0154 & 0.1098 & 1.5033 \\
\hline & 200 & 0.0288 & -0.0310 & -0.0680 & 0.2712 & 0.0303 & 0.0125 & 0.0903 & 1.3002 \\
\hline & 250 & 0.0296 & -0.0312 & -0.0715 & 0.2479 & 0.0286 & 0.0107 & 0.0769 & 1.1736 \\
\hline & 300 & 0.0298 & -0.0247 & -0.0580 & 0.2067 & 0.0269 & 0.0094 & 0.0693 & 1.0463 \\
\hline & 350 & 0.0299 & -0.0267 & -0.0650 & 0.1994 & 0.0257 & 0.0084 & 0.0627 & 0.9814 \\
\hline & 400 & 0.0351 & -0.0239 & -0.0558 & 0.1540 & 0.0252 & 0.0075 & 0.0568 & 0.8998 \\
\hline & 450 & 0.0312 & -0.0226 & -0.0563 & 0.1611 & 0.0244 & 0.0070 & 0.0534 & 0.8452 \\
\hline & 500 & 0.0315 & -0.0218 & -0.0524 & 0.1426 & 0.0234 & 0.0064 & 0.0496 & 0.7979 \\
\hline \multirow{10}{*}{ S3 } & 50 & -0.0550 & $\begin{array}{l}-0.0038 \\
\end{array}$ & 0.0441 & 0.4134 & $\begin{array}{l}0.1099 \\
\end{array}$ & 0.0211 & 0.3530 & 1.3615 \\
\hline & 100 & -0.0554 & -0.0259 & -0.0733 & 0.5147 & 0.1103 & 0.0118 & 0.1407 & 1.5007 \\
\hline & 150 & -0.0557 & -0.0304 & -0.0963 & 0.5292 & 0.1077 & 0.0090 & 0.1087 & 1.5471 \\
\hline & 200 & -0.0497 & -0.0343 & -0.1143 & 0.5316 & 0.1120 & 0.0078 & 0.0953 & 1.5011 \\
\hline & 250 & -0.0515 & -0.0334 & -0.1114 & 0.4953 & 0.0983 & 0.0065 & 0.0804 & 1.3354 \\
\hline & 300 & -0.0516 & -0.0309 & -0.1070 & 0.4685 & 0.0912 & 0.0056 & 0.0703 & 1.2109 \\
\hline & 350 & -0.0527 & -0.0298 & -0.1036 & 0.4514 & 0.0854 & 0.0051 & 0.0653 & 1.1433 \\
\hline & 400 & -0.0440 & -0.0281 & -0.0977 & 0.4093 & 0.0815 & 0.0046 & 0.0575 & 1.0227 \\
\hline & 450 & -0.0495 & -0.0278 & -0.0990 & 0.4156 & 0.0793 & 0.0042 & 0.0544 & 0.9910 \\
\hline & 500 & -0.0478 & -0.0260 & -0.0963 & 0.4008 & 0.0776 & 0.0038 & 0.0500 & 0.9252 \\
\hline \multirow{10}{*}{ S4 } & 50 & -0.0273 & 0.0083 & 0.0505 & 0.3114 & 0.0348 & 0.1012 & 0.1911 & 0.8594 \\
\hline & 100 & -0.0368 & -0.0361 & -0.0263 & 0.4095 & 0.0351 & 0.0495 & 0.0645 & 1.0115 \\
\hline & 150 & -0.0396 & -0.0493 & -0.0522 & 0.4527 & 0.0357 & 0.0352 & 0.0446 & 1.1052 \\
\hline & 200 & -0.0558 & -0.0640 & -0.0726 & 0.5436 & 0.0344 & 0.0300 & 0.0396 & 1.2789 \\
\hline & 250 & -0.0575 & -0.0625 & -0.0724 & 0.5297 & 0.0317 & 0.0263 & 0.0354 & 1.2056 \\
\hline & 300 & -0.0537 & -0.0643 & -0.0760 & 0.5109 & 0.0313 & 0.0229 & 0.0315 & 1.1608 \\
\hline & 350 & -0.0519 & -0.0652 & -0.0777 & 0.4994 & 0.0303 & 0.0205 & 0.0288 & 1.1263 \\
\hline & 400 & -0.0472 & -0.0595 & -0.0711 & 0.4575 & 0.0290 & 0.0185 & 0.0262 & 1.0081 \\
\hline & 450 & -0.0493 & -0.0598 & -0.0707 & 0.4487 & 0.0275 & 0.0169 & 0.0241 & 0.9463 \\
\hline & 500 & -0.0470 & -0.0559 & -0.0680 & 0.4355 & 0.0275 & 0.0156 & 0.0226 & 0.9184 \\
\hline
\end{tabular}

\section{Real Data Applications}

In this section, five real data applications for the GFW distribution are applied. GFW distribution is fitted to the real data sets estimating the parameter using five methods of estimation given in Section 2. The MLE, LSE, WLSE, ADE and CVME of the parameters of GFW distribution are also obtained by BFGS algorithm and reported in Tables 6-8. Also, the 
standard errors based on MLE of all parameters for five real data sets are given, respectively, by $(0.4394,1.9314,33.0842,6.2633),(0.0488,0.9051,0.0860,2.6563),(0.0938,0.8325$, $1.1973,3.1906),(0.2339,2.7112,0.1207,0.8655)$ and $(0.5331,7.9961,7.1098,6.9551)$.

In the following we give the five real data sets;

Data Set 1 (Kundu and Raqap, 2009)

$1.312,1.314,1.479,1.552,1.700,1.803,1.861,1.865,1.944,1.958,1.966,1.997,2.006,2.021,2.027,2.055,2.063,2.098$, $2.140,2.179,2.224,2.240,2.253,2.270,2.272,2.274,2.301,2.301,2.359,2.382,2.382,2.426,2.434,2.435,2.478,2.490$, $2.511,2.514,2.535,2.554,2.566,2.570,2.586,2.629,2.633,2.642,2.648,2.684,2.697,2.726,2.770,2.773,2.800,2.809$, $2.818,2.821,2.848,2.880,2.809,2.818,2.821,2.848,2.880,2.954,3.012,3.067,3.084,3.090,3.096,3.128,3.233,3.433$, $3.585,3.585$.

Data Set 2 (Nichols and Padgett, 2006)

$0.39,0.85,1.08,1.25,1.47,1.57,1.61,1.61,1.69,1.80,1.84,1.87,1.89,2.03,2.03,2.05,2.12,2.35,2.41,2.43$, $2.48,2.50,2.53,2.55,2.55,2.56,2.59,2.67,2.73,2.74,2.79,2.81,2.82,2.85,2.87,2.88,2.93,2.95,2.96,2.97$, $3.09,3.11,3.11,3.15,3.15,3.19,3.22,3.22,3.27,3.28,3.31,3.31,3.33,3.39,3.39,3.56,3.60,3.65,3.68,3.70$, $3.75,4.20,4.38,4.42,4.70,4.90$.

Data Set 3 (Bjerkedal, 1960)

$0.77,1.74,0.81,1.20,1.95,1.20,0.47,1.43,3.37,2.20,3.00,3.09,1.51,2.10,0.52,1.62,1.31,0.32,0.59,0.81$, $2.81,1.87,1.18,1.35,4.75,2.48,0.96,1.89,0.90,2.05$

Data Set 4 (Xu et al., 2003)

$1.6,3.5,4.8,5.4,6.0,6.5,7.0,7.3,7.7,8.0,8.4,2.0,3.9,5.0,5.6,6.1,6.5,7.1,7.3,7.8,8.1,8.4,2.6,4.5,5.1,5.8,6.3,6.7$, 7.3, 7.7, 7.9, 8.3, 8.5, 3.0, 4.6, 5.3, 6.0, 8.7, 8.8, 9.0.

Data Set 5 (Lawless, 1982)

2.836, 3.120, 3.045, 5.169, 4.934, 4.970, 3.018, 3.770, 5.272, 3.856, 2.046

Table 6: Parameter estimation for all real data sets based on the MLE and LSE

\begin{tabular}{lccccccccc}
\hline \multicolumn{1}{c}{ MLE } \\
\hline $\begin{array}{l}\text { Data } \\
\text { set }\end{array}$ & $\alpha$ & $\beta$ & $\theta$ & $\gamma$ & $\alpha$ & $\beta$ & $\theta$ & $\gamma$ \\
\hline 1 & 0.3064 & 11.8222 & 36.6524 & 3.3635 & 0.2788 & 12.1092 & 42.2601 & 6.1689 \\
2 & 0.0439 & 1.6871 & 0.0497 & 7.6163 & 0.1863 & 1.7785 & 0.0993 & 3.6235 \\
3 & 0.0548 & 1.2928 & 0.7054 & 3.1252 & 0.0576 & 0.8448 & 0.2734 & 3.8642 \\
4 & 0.2927 & 3.9180 & 0.1002 & 0.2664 & 0.2978 & 3.3609 & 0.1064 & 0.1805 \\
5 & 0.2960 & 8.1233 & 1.3459 & 1.1444 & 0.1757 & 7.9264 & 2.8524 & 0.3722 \\
\hline
\end{tabular}

Table 7: Parameter estimation for all real data sets based on the WLSE and ADE

\begin{tabular}{lccccccccc}
\hline \multicolumn{1}{c}{ WLSE } \\
$\begin{array}{l}\text { Data } \\
\text { set }\end{array}$ & $\alpha$ & $\beta$ & $\theta$ & $\gamma$ & $\alpha$ & $\beta$ & $\theta$ & $\gamma$ \\
\hline 1 & 0.1863 & 11.2302 & 33.5804 & 16.5374 & 0.3661 & 11.8586 & 31.5820 & 2.5584 \\
2 & 0.1129 & 1.5883 & 0.0559 & 5.5188 & 0.0258 & 2.4123 & 0.1320 & 8.4569 \\
3 & 0.4331 & 0.7626 & 0.2042 & 4.3505 & 0.0389 & 0.9493 & 0.3127 & 4.1959 \\
4 & 0.3114 & 2.5088 & 0.0796 & 0.1636 & 0.2649 & 3.6030 & 0.1129 & 0.3268 \\
5 & 0.1797 & 7.8625 & 2.5029 & 0.9826 & 0.1829 & 10.0765 & 5.1078 & 0.0233 \\
\hline
\end{tabular}


Table 8: Parameter estimation for all real data sets based on the CVME

\begin{tabular}{lclll}
\hline \multicolumn{5}{c}{ CVME } \\
\hline Data set & $\alpha$ & \multicolumn{1}{c}{$\beta$} & \multicolumn{1}{c}{$\theta$} & $\gamma$ \\
\hline 1 & 0.3014 & 12.2587 & 42.2299 & 6.2834 \\
2 & 0.1815 & 2.0880 & 0.1296 & 3.8467 \\
3 & 0.0619 & 1.0264 & 0.39911 & 3.6164 \\
4 & 0.3007 & 3.9545 & 0.1159 & 0.1874 \\
5 & 0.2428 & 8.4646 & 2.6545 & 0.0595 \\
\hline
\end{tabular}

\section{Concluding Remark}

In this paper, GFW distribution introduced by Khaleel et al. (2020) is examined in views of some point estimations. Five estimators are studied to estimate the four parameters of GFW distribution. A new extension is ensured for the estimation of the parameters for GFW distribution. Monte Carlo simulations are conducted for different parameter values and different sample sizes. It is concluded that as sizes of samples increases, the biases and especially MSEs of all estimators decreases and close to zero. In conclusion, we recommend the MLE and WLSE to estimate the parameters of GFW distribution due to they generally have smallest MSE according to simulation results. The parameter estimates of GFW distribution are obtained using five different estimation methods for five practical data sets. In future studies, the other estimation methods such as percentile, Bayes, moments methods, etc. can be discussed for point estimation of parameters of the GFW distribution.

\section{References}

Ali, S., Dey, S., Tahir, M. H., Mansoor, M. (2020a). Two-Parameter Logistic-Exponential Distribution: Some New Properties and Estimation Methods. American Journal of Mathematical and Management Sciences, 39(3), 270-298.

Ali, S., Dey, S., Tahir, M., Mansoor, M., (2020b). "The Comparison Of Different Estimation Methods For The Parameters Of Flexible Weibull Distribution", Communications Series A1 Mathematics \& Statistics, 69, 794-814.

Anderson, T. W., Darling, D. A., (1952). "Asymptotic theory of certain goodness of fit criteria based on stochastic processes", The Annals of mathematical statistics, 23(2), 193-212.

Asgharzadeh, A., Rezaie, R., Abdi, M., (2011). "Comparisons of methods of estimation for the half-logistic distribution”, Selçuk Journal of Applied Mathematics, 93-108.

Bebbington, M., Lai, C. D., Zitikis, R., (2007). "A flexible Weibull extension," "Reliability Engineering \& System Safety", 92, 719-726.

Bjerkedal, T., (1960). “Acquisition of Resistance in Guinea Piesinfected with Different Doses of Virulent Tubercle Bacill”, American Journal of Hygiene, 72, 130-148.

Dey, S., Ali, S., Kumar, D. (2020). Weighted inverted Weibull distribution: Properties and estimation. Journal of Statistics and Management Systems, 23(5), 843-885. 
Eliwa, M. S., El-Morshedy, M., Ali, S. (2020). Exponentiated odd Chen-G family of distributions: statistical properties, Bayesian and non-Bayesian estimation with applications. Journal of Applied Statistics, 1-27.

Karakaya, K., Tanış, C. (2020a) Different methods of estimation for the one parameter Akash distribution. Cumhuriyet Science Journal, 41(4), 944-950.

Karakaya, K., Tanış, C. (2020b) Estimating the Parameters of Xgamma Weibull Distribution. Adlyaman Üniversitesi Fen Bilimleri Dergisi, 10(2), 557-571.

Khaleel, M. A., Oguntunde,, P. E., Ahmed,, M.T., Ibrahim, N.A., Loh Y.F., (2020). "The Gompertz Flexible Weibull Distribution and its Applications," Malaysian Journal of Mathematical Sciences, 14, 169-190.

Kundu, D., Raqab, M. Z., (2009). "Estimation of $\mathrm{R}=\mathrm{P}(\mathrm{Y}<\mathrm{X})$ for three parameter Weibull distribution”, Stat. Probab. Lett., 79, 1839-1846.

Kurban, M., Kanta, Y., Hocaoğlu, F. O., (2007). "Weibull Dagılımı Kullanılarak Rüzgar Hız ve Güç Yoğunluklarının İstatistiksel Analizi”, Afyon Kocatepe Üniversitesi Fen Ve Mühendislik Bilimleri Dergisi, 7, 205-218.

Lawless, J. F., (1982). "Statistical Models and Methods for Lifetime Data", Second ed., Wiley, New York.

Macdonald, P., (1971). "Comments and Queries Comment on An Estimation Procedure for Mixtures of Distributions by Choi and Bulgren", Journal of the Royal Statistical Society: Series B (Methodological), 33 (2), 326-329.

Nassar, M., Afify, A. Z., Dey, S., Kumar, D., (2018). “A new extension of Weibull distribution: properties and different methods of estimation", Journal of Computational and Applied Mathematics, 336, 439-457.

Nichols, M. D., Padgett, W. J., (2006). “A bootstrap control chart for Weibull percentiles”, Quality and Reliability Engineering International, 22, 141-151.

Peng, X., Yan, Z., (2014). "Estimation and application for a new extended Weibull distribution", Reliability Engineering \& System Safety, 121, 34-42.

Swain J.J., Venkatraman S., Wilson, J. R., (1988). "Least-square estimation of distribution functions in johnson's translation system”, J. Stat. Comput. Simul., 29, 271-297.

Taniş, C., Saracoglu, B., (2019). "Comparisons of six different estimation methods for logKumaraswamy distribution”, Thermal Science, 23, 1839-1847.

Tanış, C. (2021) On Transmuted Power Function Distribution: Characterization, Risk Measures, and Estimation. Journal of New Theory, (34), 72-81. 
Xu, K. Xie, M., Tang, L. C., Ho, S. L., (2003). “Application of neural networks in forecasting engine systems reliability”, Applied Soft Computing, 2, 255-268. 